\title{
ESQUISSE DU PARLEMENTARISME ET DU MONOPARTISME EN AFRIQUE: LE CAS DU TOGO
}

\author{
Emile A.B. VAN ROUVEROY VAN NIEUWAAL \\ Afrika-Studiecentrum \\ Postbus 9507 \\ 2300 RA Leiden \\ Pays-Bas
}

CHAMPS D'INVESTIGATION: Pouvoir traditionnel et constitutionnalisme en Afrique

\section{SUMMARY}

\section{AN OUTLINE OF THE PARLIAMENTARISM AND THE ONE PARTY SYSTEM IN AFRIKA: THE CASE OF TOGO.}

Witnessed the last hundred years profound political and constitutional changes. In this respect there are many differences between African States depending on which kind of colonial overlord has been the ruling power. The African State was a juridical entity in international law, but was it also, at the time of independence, an empirical entity in national fact?

In almost all cases the empirical reality as a functioning government was still primarily the presence of European bureaucrats who has embodied the colonial state. Independence therefore opened a gap between the international legitimacy and the internal marginality of many emergent African State. The gap often presented a real political dilemma to the new African rulers: they usually could retain European officials only by compromising their national independence and could dispense with them only at the risk of undermining governmental performance. 
After adoption of European constitutional law and with, initially, a high degree of similarity between most constitutions in French-speaking Africa, nowadays these constitutions differ widely, contrary to the Commonwealth Africa that has experienced fewer innovations and constitutional breakdowns. Parliamentary systems of government gave way to One Party Systems, introduced in many cases by the national army, as the best equiped, trained, paid and organised power in the country.

This has been the political and constitutional development in Togo as well. Since 1969, it has a One Party System, the Rassemblement du Peuple Togolais, founded by the President of the Togolese Republic, Gen. Gnassingbé Eyadéma. But, as everywhere else, doubts are growing about the effectiveness of the One Party System. Are the Togolese perspectives such that Eyadéma is willing to have an open mind and to be all ears for the critics to his own creation? Another intriguing question is to know in which way the African traditional authorities ("chieftancy"), as the core of the concern for the local world and as the embodiment of a moral and political order, could have a new (?) role in the socio-political development of the state in Africa? Will the chiefs' position in the long term be that of a noble élite, an echo from the past, useful as a tourist attraction or as managers of fun parks with safari possibilities.

KEY WORDS: chieftancy, constitutions, legal pluralism, one party system, politics, Togo.

\section{INTRODUCTION}

Une des descriptions les plus vivantes de l'appareil constitutionnel et politique africain nous est offerte par Hesseling (1985: 15), lorsqu'elle nous promène, a Dakar, le long des bâtiments qui abritent les organes gouvernementaux du Sénégal, cette ancienne colonie française:" ..., le Sénégal possède en effet depuis son indépendance acquise en 1960 une constitution qui prévoit la charge présidentielle. A partir de cette date et jusqu'à la fin 1980, Léopold Sédar Senghor assuma les fonctions de chef de l'Etat... En face du palais s'élève le Building administratif, immeuble gris de dix étages où vont travailler chaque jour de nombreux fonctionnaires... Nous quittons le bâtiment par l'avenue de la République, puis, prenant à gauche, nous nous 
dirigeons vers une construction semi-circulaire flanquée de majestueux escaliers: l'arène de l'Assemblée nationale. Depuis que les députés du Parti démocratique socialiste y firent leur entrée, en 1978, aux côtés des députés du Parti socialiste au pouvoir, le jeu parlementaire s'est considérablement animé et ravit tant les yeux que les oreilles: certains membres du PDS portent avec ostentation des boubous (caftans) de la couleur de leur parti, bleu clair, et leurs nombreuses interventions ne manquent pas de véhémence".

Une description similaire, certes légèrement modifiée, pourrait s'appliquer au centre administratif de Lomé, capitale du Togo, pays qui faisait également partie de "l'empire" colonial français en Afrique, quoique ce pays ne fut pas une colonie française, mais un pays sous tutelle française selon un mandat de la SDN. Pourtant, l'image évoquée serait rapidement différente. En effet, arrivé à la hauteur de l'Assemblée nationale, le promeneur est frappé par le silence: tandis que, depuis 1976, le Sénégal connaît un régime pluripartiste, le Togo compte depuis 1969 parmi les nombreux Etats africains monopartistes. La vie politique est dominée par le Rassemblement du Peuple Togolais, dont les organes ont leur siège à la Maison du Rassemblement du Peuple Togolais et non à l'Assemblée nationale, qui reste désertée .

Cette différence fondamentale nous montre tout de suite l'étendue et la variété du continent africain, non seulement sur le plan géographique, mais aussi sur le plan des constitutions et du droit constitutionnelle, même lorsqu'il s'agit, comme dans le cas présent, de deux Etats ayant un passé colonial commun. L'évolution des constitutions a transformé l'Afrique en un véritable caléidoscope qui s'étend de l'Egypte au royaume du Maroc et comprend les Etats de l'Afrique sub-saharienne qui portent toujours l'empreinte de leur passé français, anglais, belge, allemand, portugais ou espagnol, sans parler de la République sud-africaine, au passé bien particulier, et sans oublierl'influence de l'Union soviétique et les Etats-Unis.

L'Afrique comprend plus de 50 Etats. Il serait donc vain de prétendre en présenter toute la diversité constitutionnelle dans le cadre limité du présent article. Pour plus amples informations, le lecteur pourra consulter la bibliographie, toute modeste soit-elle. Le présent article ne pourra être exhaustif ni en ce qui concerne le thème, ni en ce qui concerne l'aspect géographique: "seuls" les pays situés au sud du Sahara et ayant un passé colonial français 


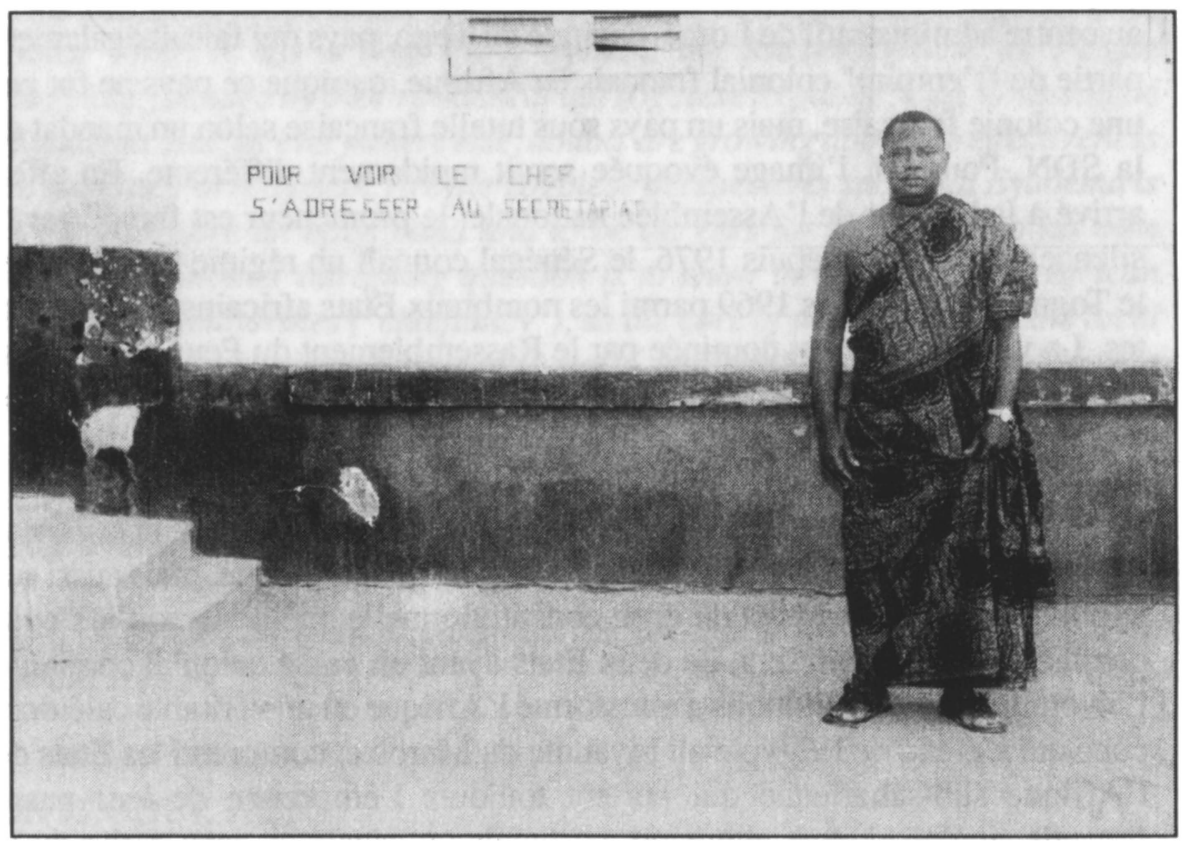

De chef de canton van Be in Lomé (1986)

Foto: EAB van Rouveroy van Nieuwaal 
ou anglais y seront traités, en particulier la République du Togo où j'effectue des recherches depuis 1969 en tant qu'anthropologue du droit.

Avant l'arrivée des Européens, la société africaine était organisée de telle manière que ses membres faisaient partie intégrante d'un réseau complexe de liens à la fois sociaux et juridiques qui étaient et sont encore, dans bien des cas, indissociables. Les liens de parenté, les rapports économiques, religieux et politiques constituaient un tout comme c'est encore le cas aujourd'hui. Il convient de garder à l'esprit cet aspect lorsqu'on entreprend l'étude du droit, constitutionnel ou autre, de l'Afrique. Les distinctions que connaissent les systèmes juridiques européens entre droit privé et droit pénal, entre droit constitutionnel et droit administratif sont totalement inconnus en droit (coutumier) africain. Actuellement, toutefois, les employeurs africains appliquent ces subdivisions du droit, suivant ainsi de façon parfaite (ou si l'on prefere: perfide) le chemin tracé par les anciennes autorités coloniales. Il est facile de comprendre cette attitude puisque le juriste africain a souvent été formé dans une université européenne ou bien a étudié le droit dans une des universités africaines qui offrent toujours une place prépondérante à l'étude du droit européen. Il va de soi que cette situation précoloniale et coloniale a subi de profonds changements par suite de l'arrivée des autorités coloniales, de l'introduction de l'islam et de la religion chrétienne, de la mise en place de nouveaux systèmes sociaux-économiques tels que le capitalisme, le socialisme et le marxisme. Non seulement tous ces facteurs externes ont exercé une influence sur le droit constitutionnel non-écrit africain et du droit coutumier, mais ils ont également entraîné l'apparition, dans les pays africains, de différents systèmes juridiques simultanément en vigueur.

On parle dans ce cas de pluralisme juridique, terme par lequel il faut entendre que des personnes, formant un groupe constitué par des autorités coloniales ou bien au hasard d'une politique nationale, sont soumises à un ensemble de règles de droit qui, au lieu de découler d'un modèle socio-culturel plus ou moins homogène, proviennent de sociétés aux structures différentes, mues par des mentalités et des objectifs opposés. Ce pluralisme juridique ne se limite pas au droit coutumier "civil". Le droit constitutionnel se caractérise aussi par une multitude d'appareils juridiques. En effet, avant l'arrivée des Européens, l'Afrique connaissait un grand nombre d'appareils constitutionnels traditionnels qui différaient considérablement les uns des autres dans les détails, comme ce fut d'ailleurs le cas plus tard des systèmes nationaux qui 
se succédèrent à un rythme accéléré, par exemple au Ghana. Il en est résulté de multiples interactions. Mais, plus important encore, la diversité du droit coutumier a rendue vaine, dès le départ, toute tentative de concevoir un seul système juridique, fondé sur le droit coutumier (Goldschmidt, 1984: 31). Et, bien sûr, ce phénomène s'applique également au niveau du pluralisme juridique constitutionnel.

Mais il est un aspect du pluralisme juridique qui revêt un caractère particulièrement important, à savoir le fait que des règles de droit, qui, quant à la forme et au contenu étaient empruntées en partie ou en totalité au droit européen, ont été ajoutées au droit africain, en raison du développement socio-économique ou politique, mais qui ne correspondent nullement aux conceptions juridiques de la population africaine. Pratiquement, tout Etat africain a traversé, dans son passé colonial, des phases similaires. Et jusqu'a nos jours l'Etat africain se trouve dans la même situation, car, de même que ses populations, il entretient des liens socio-culturels, politiques et économiques, de plus en plus étroits avec les Etats non-africains.

Bien que je ne puisse pas entamer dans ce contexte les problèms juridiques nombreux qui se présentent aux pays africains depuis la réalisation de leur indépendance, sous un seul point de vue les idées des législateurs en Afrique semblaient être de la même teneur. C'était l'idée que la diversité du droit, agrandie encore par le législateur colonial par l'importation de son propre système juridique, aura à ceder la place à un droit national unifié dans l'avenir proche (van Rouveroy van Nieuwaal, 1976: 230). Ici on partait de la conception que diversité de droit et sous-développement allaient (ou vont toujours?) de pair, conception qui trouvait dans l'occident aussi des adhérents (Trubek, 1972). Le législateur caressait l'espoir qu'un seul système de droit était favorable à l'évolution économique et sociale du pays. On craignait que le pluralisme juridique empéchait le développement économique et social du pays. On craignait que le pluralisme juridique empéchait le développement de l'économie, tandis que du point de vue social il était pourvu aux besoins de diverses figures juridiques (comme la hypothèque, les assurances, la législation sociale, etc.) auxquelles le droit coutumier ne pourvoyait pas. Le droit unifié devrait alors servir d'outil au moyen duquel des buts sociaux divers pouvaient être réalisés. Mais les efforts entrepris pour unifier le droit s'expliquaient également par le fait que celui-ci constituait un instrument de choix grâce auquel une élite dominante visait à s'imposer en tant avant-garde 
du progrès et détentrice de la constitution idéale. Enfin, il faut bien se rendre compte qu'un seul système juridique (constitutionnel) national est un mécanisme que l'on peut actionner pour répartir ou centraliser le pouvoir, ou bien pour renforcer et légitimer l'autorité d'une petite élite. Dans une société fortement stratifiée, l'élite a intérêt à lutter pour l'homogénéité, d'autant plus que la culture de cette élite, ou du moins le comportement auquel elle s'est conformée, sert de norme permettant de définir cette homogénéité.

\section{L'ETAT EN AFRIQUE}

L'Etat africain a connu une véritable mutation au cours des cent dernières années. L'histoire constitutionnelle de l'Afrique nous offre une succession de structures différentes et de formes multiples du pouvoir: des formes historiques, telles que les systèmes segmentaires, les petites unités autonomes, les royaumes tributaires, etc, en passant par diverses versions de l'état colonial et par des mouvements inspirés par l'indépendance, aux démocraties plus ou moins parlementaires, par exemple au Sénégal (depuis 1976), Nigéria (entre 1979 et 1984) et Ghana (entre 1969 et 1979), et aux régimes monopartistes (Togo, Guinée, Tanzanie, Gabon, Malawi, Sierra Léone, Zambie et Zaïre), ou finalement militaires et dictatoriaux.

Il est incontestable que l'Afrique comme tout autre continent d'ailleurs, est constamment le thếtre de changements, qu'ils soient politiques, économiques ou sociaux. Les moyens de communication de masse y ont fait leur entrée, de nombreux liens socio-économiques sont apparus entre la population citadine et la population rurale. Certaines sociétés, qui vivaient traditionnellement en autarcie, ont vu leur agriculture et leur élevage se commercialiser et se mécaniser. L'Etat est plus ou moins omniprésent surtout dans les pays à petite échelle comme le Togo, et le citoyen africain doit encore s'habituer au fait qu'il semble dépendre de plus en plus de lui pour accéder aux sources du bien-être social. Il s'agit ici d'une évolution, déjà perceptible à l'époque coloniale, qui s'est poursuivie depuis à un rythme accéléré. Toutefois, il ne faudrait pas croire que la relation Etat-citoyen soit maintenant la même en Afrique qu'en Europe. Tandis qu'en Occident, le développement du capitalisme, du néo-capitalisme et du socialisme a permis de mettre en place une nouvelle société bourgeoise, en Afrique, l'introduction du capitalisme et d'autres systèmes économiques et politiques a rompu l'hégémonie 


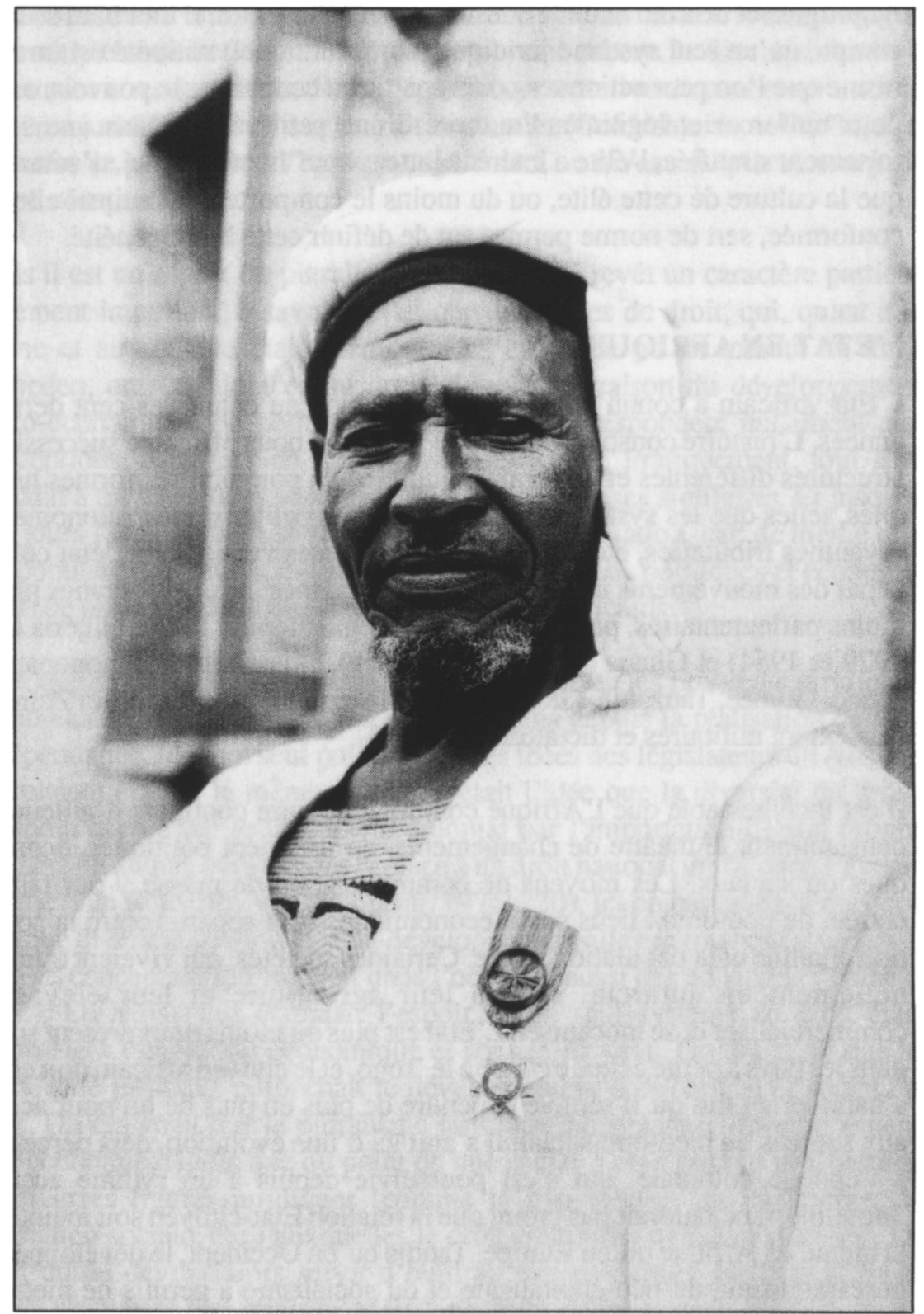

Na Tyaba Tyekura, chef supérieur van de Anufom (1963-1978) in Noord-Togo, na zijn aanstelling tot ridder van de Orde van Mono

Foto: EA.B. van Rouveroy van Nieuwaal 
régnante. Une nouvelle élite économique et politique est apparue, mais, en même temps, "l'ancienne" élite des chefs coutumiers s'est partiellement maintenue, notamment des chefs de famille qui ont plus ou moins su maintenir leur autorité par exemple à l'occasion des mariages en exigeant des prestations matrimoniales.

Dans certains Etats africains, la constitution a par mesures diverses, éliminé la chefferie coutumière, ou l' autorité "neo- traditionnelle" pour reprendre mes propres termes (van Rouveroy van Nieuwaal, 1988), même si, à mon avis, il ne faut pas aller jusqu'à dire que cette institution a été supprimée de la société. Je ne donne qu'un seul exemple: la Tanzanie ou, en 1963, une loi abolissait la chefferie, et mit ainsi un terme à un processus qui s'était amorcé vers la fin de la Deuxième Guerre mondiale. Depuis des années, la législation visait à saper le pouvoir des chefs coutumiers qui revenait de plus en plus souvent aux cadres du parti unitaire, le TANU. Ces cadres n'appartenaient pas à la famille des chefs coutumiers. Toutefois, il ne faut pas penser que ces réformes juridiques aient porté atteinte aux privilèges socio-économiques dont jouissaient les familles de chefs, surtout depuis la période coloniale. En effet, les ex-chefs coutumiers avaient accumulé sufisamment de richesses pour permettre à leurs descendants d'étudier dans les meilleures écoles. Par la suite, quand apparut un besoin aigu de personnel qualifie, ces descendants purent se caser dans les diverses institutions administratives et juridiques et réussirent ainsi à pénétrer au coeur même du monde de la politique et des affaires (Moore, 1987: 75).

La place qu'occupe la constitution africaine actuelle par rapport au droit national mérite une étude particulière. J'expliquerai cette assertion à l'aide d'un seul exemple: au Rhodésie (Zimbabwe), dernier bastion de l'empire colonial britannique en Afrique, le gouvernement du Président actuel, Mugabe, se mit au travail en 1980 en se basant sur une constitution "drafted and given by the British, which the Prime Minister scarcely had chance to glance at between its being laid before the United Kingdom Parliament on December 7, 1979". La constitution du Zimbabwe datant de 1979 peut donc difficilement être qualifiée de document national. Cette constitution contient un certain nombre de dispositions concernant les relations entre le président de la République, le premier ministre, les autres ministres et le parlement qui sont plus ou moins considérées comme une codification du régime parlementaire britannique (étant bien entendu que les 20 sièges destinés à la minorité 
blanche ne sont naturellement pas empruntés à la constitution britannique). Cependant, la vie politique est davantage régie par les dispositions relatives à l'état d'exception que par la codification de l'appareil parlementaire.

Cette situation, d'ailleurs, ne s'observe pas qu'au Zimbabwe. Dans de nombreux pays en voie de développement, l'état d'exception manié par le pouvoir exécutif a beaucoup plus de poids que les principes empruntés au droit constitutionnel anglais ou français, en vertu desquels le pouvoir exécutif est tenu de respecter la loi, et une loi doit recevoir l'assentiment du parlement.

Pour clore ce point, je voudrais faire une dernière observation sur l'Etat africain. Au moment de l'indépendance, l'Etat africain était bel et bien une réalité juridique de droit international, mais cela signifie-t-il qu'il existait une réalité empirique au niveau national (Jackson \& Rosberg, 1985:46)? Dans de nombreux cas, en effet, l'Etat colonial était conduit et soutenu par des fonctionnaires des administrations européennes qui retournèrent chez eux après l'indépendance, mettant en danger le fonctionnement de l'Etat en tant qu'unité politico- administrative. Les Etats ne disposaient généralement pas d'un nombre suffisant de cadres africains formés pour occuper les postes laissés vacants. Les responsables se trouvèrent ainsi devant un dilemme: "They usually could retain European officials only by compromising their national independence and could dispense with them only at the risk of undermining governmental performance "(Jackson \& Rosberg, 1985:46).

Un des exemples les plus tristes de désintégration de l'appareil administratif nous fut offert par le Congo belge, le Zaïre, qui après une mutinerie des troupes africaines à la fin du mois de juin en 1960, devint pratiquement ingouvernable lorsque les Belges, craignant pour leur vie, quittèrent en masse le pays. On remplaça rapidement la constitution de 1960 qui était, à peu de choses, prèsqu'une copie de la constitution belge. Certes, la constitution de 1964 renforça le caractère fédéral de l'Etat du Zaïre et étendit les pouvoirs de l'exécutif au détriment du Parlement, mais il était trop tard pour empêcher le chaos. Telle était la situation qui poussa Mobutu à prendre le pouvoir en 1965.

L'évolution politique et constitutionnelle des Etats africains se caractérise par trois phénomènes principaux: l'apparition d'un pouvoir exécutif présidentiel fort, la mise en place de régimes monopartistes, et une grande instabilité due 
aux coups d'Etat militaires qui se succèdent rapidement, créant régulièrement des vides constitutionnels. Comment est-on arrivé à cette situation?

L'Etat colonial était un Etat exécutif dans lequel les pouvoirs étaient fortement concentrés entre les mains du Gouverneur, le pouvoir suprême de la puissance coloniale sur place. Ce dernier revêtait non seulement le pouvoir exécutif, mais aussi une bonne partie du pouvoir législatif et judiciaire. Au moment de l'indépendance, les Etats africains reçurent en héritage des structures administratives centralisées et quasi-militaires (Coleman \& Rosberg, 1971). Pourtant, les nouvelles constitutions introduisirent dans les nouveaux Etats des appareils parlementaires inspirés du modèle anglais de Westminister ou de la constitution française. Ces derniers sont imprégnés de la théorie de "la trias politica", des droits civiques et de la défense des minorités, pour ne citer que certains aspects. Là où la structure de l'administration coloniale reposait sur un pouvoir non-partagé, les Etats africains durent, sans aucune transition, fonctionner comme des démocraties européennes. Il n'est nullement surprenant qu'ils n'y soient pas parvenus. Le centralisme bureaucratique colonial avait duré trop longtemps pour que cela fût encore possible, et avait en outre empêché la mise en place d'autres usages, d'avantage inspirés de la démocratie et du parlementarisme. Ainsi pourrait s'expliquer l'apparition de régimes monopartistes au pouvoir présidentiel ferme et au pouvoir exécutif fort.

\section{EVOLUTION CONSTITUTIONNELLE ET POLITIQUE DEPUIS LES ANNÉES 1950}

Ce n'est que dans les années cinquante que les puissances coloniales tentèrent d'introduire des systèmes démocratiques d'administration. Les Français appliquèrent dans leurs territoires la loi du 23 juin 1956, connue sous le nom de "loi- cadre", en vertu de laquelle des assemblées consultatives furent élues au suffrage universel, tandis que, quelques années avant l'indépendance, les britanniques expérimentèrent dans leurs territoires avec de nouvelles structures d'administration interne autonome (Sklar, 1985:7), dans lesquelles la participation des chefs coutumiers fut très vivement critiqué par la nouvelle élite politique (van Rouveroy van Nieuwaal, 1988: 73; Sklar, 1985: 8). Malgré ces mesures préparatoires, récentes et superficielles, la plupart des Etats africains avaient, au moment de l'indépendance, des constitutions de type 
parlementaire et un appareil pluripartiste qui rendait le gouvernement responsable devant le Parlement. Mais cette situation a été de courte durée.

Dans la période qui a suivi la proclamation de l'indépendance, il était assez facile de classer les constitutions africaines: les colonies anglaises basèrent leur constitution sur ce que l'on a appelé le modèle de Westminister, qui avait été établi lors des conférences de Lancaster House, tandis que les anciens territoires français s'inspirèrent de la constitution française de 1958, à l'exception toutefois de la République de Guinée. Ce pays disait expressément "non" au référéndum demandant s'il voulait être admis dans la Communauté française que la France s'apprêtait à créer en Afrique avec ses anciens territoires. Les conséquences furent désastreuses pour la Guinée: dans un délai d'un mois, tous les Français partirent, emportant tout avec eux, jusqu'aux appareils téléphoniques arrachés aux maisons et aux bureaux. Du jour au lendemain, le désordre régna dans le pays (Hesseling, 1985:172).

L'ancien Congo belge accueillit l'indépendance avec une constitution que le Parlement belge avait pratiquement copiée sur la constitution belge et le lui laissat, "prête à l'emploi".

On peut donc constater que la réception du droit constitutionnel européen fut considérable. Fernandes Mendes (1989:114) en a encore exposé récemment les raisons, dont une des plus importantes était le désir des anciennes "mèrepatries" d'entretenir des relations économiques avec leurs anciennes colonies. Une constitution fondée sur "la trais politica", les droits fondamentaux en vigueur en Europe et des élections libres pouvait les y aider.

Cependant, les choses ont bien changé depuis: de 1960 à 1985, 43 constitutions ont vue le jour dans 18 anciennes possessions françaises, ce qui revient a 2,4 constitutions par Etat. Cette avalanche de constitutions a rompu l'uniformité originale. L'Afrique du Commomwealth connut une évolution différente: non seulement l'élimination des constitutions originales en faveur de nouvelles y fut passablement faible, mais en outre ces nouvelles constitutions montraient de nombreux points communs avec les précédentes (Reyntjens, 1986:75).

Sous la forte influence (personnelle) de de Gaulle, il se produisit en Afrique francophone un courant, le présidentialisme, qui se caractérisait par le fait 
que le Président de la République, à la fois chef de l'Etat et chef du gouvernement, acquérait un pouvoir exceptionnel reposant sur un parti politique dominant dont il était également le chef. Cela entraîna l'adoption d'appareils monopartistes dès la cinquième ou la sixième année après la proclamation de l'indépendance: en 1966, toutes les anciennes colonies françaises avaient opté pour ce régime.

Elles ne furent pas les seules à suivre cette ligne: des régimes monopartistes apparurent également dans les anciens territoires britanniques. Le parti unique s'empara du pouvoir dans tous les organes de l'Etat: le corps législatif, le pouvoir exécutif et l'administration centrale. Ainsi, lorsqu'en 1984, Robert Mugabe annonça, au cours d'un congrès du parti dominant, le ZANU, qu'il souhaitait mettre en place un régime à parti unique, il suivait la tendance générale. Il agissait en fidèle successeur de Kwame Nkrumah qui, dans le temps (1957-1966), avait tranformé le Ghana en Etat monopartiste. La décision de Mugabe s'explique d'autant mieux qu'il avait été pendant des années un ami personnel du premier Président du Ghana.

Le nombre croissant des appareils monopartistes dominant la scène politique de leur propre style et de leur leadership personnel - souvent accompagné d'un culte de la personnalité fit également apparaître une idéologie spécifique. Voici ce que Luckman écrit:

"The single-party system reflected a basic consensus of opinion around the task of national reconstruction. In the anti-colonial period the mass nationalist party had been an expression of the united struggle against colonialism. In the post-colonial era, once the colonialist had gone, there was no remaining division between rulers and ruled, and therefore no need for conflicting parties.

Provision in the political system for a formal "opposition" would have meant straining to find a real basis of opposition for the sake of preserving an imported model of democracy. Therefore, it was agreed, the parties should attempt to retain their pre- independence character of broadly-based national movements, representative of all shades of opinion. The multi-party system was repudiated as open to manipulation and misuse of regional or tribal interest by neo-colonialist pressure". (1988:55). 
Vers la fin de la première décennie qui a suivi la proclamation de l'indépendance, le monopartisme s'était donc généralisé, même s'il montrait des différences d'un Etat à l'autre. Certain partis uniques visent uniquement la classe supérieure de la population, tandis que d'autres s'affichent ouvertement en tant que mouvements de masse. Le parti zairois devient automatiquement un mouvement de masse. Le Rassemblement du Peuple Togolais, cité plus haut, présente le même caractère, bien qu'au Togo on ne soit pas allé jusqu'd accorder automatiquement la qualité de membre à tout Togolais venant au monde.

L'Etat monopartiste se caractérise principalement par la position monopolistique, permanente et stabilisée, de fait ou de droit, d'un seul parti.

Cette description comprend aussi bien les régimes dans lesquels la loi ou la constitution n'admettent qu'un seul parti, que ceux dans lesquels un même parti occupe tous les sièges parlementaires et est en mesure d'éliminer les autres partis. Peu importe l'existence d'une opposition extra-parlementaire dans le cadre d'un appareil monopartiste de fait, puisqu'un Etat monopartiste ne se qualifie pas tant par le nombre de partis existants, que par le nombre de partis pouvant participer à la lutte pour le pouvoir, dans le cadre des règles en vigueur dans un système donné (Lavroff, 1976: 75-76).

Un courant, apparu au cours des années 70, fit une brèche dans ce monopartisme. Au Sénégal, la constitution offrit en 1976 la possibilité de mettre en place un régime pluripartiste, au début limité à trois partis, mais actuellement 19 partis politiques sont actifs. De 1977 à 1980, le Burkina Faso (qui était encore Haute Volta à l'époque) s'efforça de suivre l'exemple sénégalais, et la République Centrafricaine autorisa le pluripartisme après la chute de l'empereur Bokassa (mais le coup d'Etat militaire de 1981 mit un terme à cette experience). En revanche, le Botswana, la Gambie et, en partie, le Zimbabwe restèrent fidèles au pluri-partisme. Il règne au Botswana un climat liberal qui permet à plusieurs groupements politiques de participer au jeu du pouvoir (Hutcheson, 1988).

Si depuis 1960 l'uniformité constitutionnelle de l'Afrique francophone a fait place à une grande diversité, il n'en va pas de même en Afrique anglophone. Bien que les récentes constitutions du Zimbabwe (1980), de la Tanzanie 
(1977) et de la Sierra Léone (1978) contiennent des éléments nouveaux, elles sont encore fortement inspirées du modèle de Westminster :

"Indeed, innovations such as the recognition of one party government, the institution of provisions on 'Directive Principles of State Policy' do not fundamentally alter the constitutional structure and the organisation of state power" (Reyntjens, 1986: 89).

Il convient toutefois de noter une exception: avec la constitution adoptée en 1979, le Nigéria s'oriente davantage vers le modèle de Washington (KirkGreen, 1988; Read, 1979). Ce faible désir de renouvellement, parmi les pays africains du Commonwealth, s'explique certainement par le fait que les constitutions instaurées après la proclamation de l'indépendance ont été moins vite remplacées que dans les pays francophones.

Cependant, il faut encore remarquer un aspect important, c'est que les constitutions de l'Afrique francophone sont beaucoup plus courtes que les constitutions de langue anglaise. Les premières sont plutôt des "manifestes politiques" (Doornbos, e.a., 1984: 43-44), qui exposent de vastes projets politiques et sociaux ouverts au débat. Les constitutions africaines de langue anglaise sont plutôt des règlements administratifs qui contiennent souvent des règles détaillés du droit, telles que les droits civiques. Elles constituent une sorte de manuel destiné à être consulté.

\section{LE RÔLE POLITIQUE DE L'ARMÉE}

Depuis les années 60, l'Afrique a été le thêâtre d'un grand nombre de coups d'Etat perpétrés par des militaires. Le record a indubitablement été remporté par l'année 1966 au cours de laquelle quatre coups d'Etat ont été courronnées de succès (Burundi, Ghana, Nigéria et Haute-Volta). L'armée intervient pour des raisons diverses: en cas de scission entre l'Etat et le pouvoir constitutionnel, l'armée est la seule institution capable de venir en aide au pouvoir désagrégé. La corruption, une crise économique ou certains intérêts ethniques que les militaires estiment mal-défendus peuvent également entraîner leur intervention, de même que les très mauvais rapports existant entre l'armée et les autorités civiles par suite de divergences de vue sur la politique à mener. 
Un coup d'Etat militaire réussi se traduit par la suppression (ou du moins la suspension) de la constitution et la dissolution des partis politiques. Mais le vide constitutionnel est assez rapidement comblé, car les militaires ont eux aussi besoin de donner une assise à leur autoritité tant sur le plan interne, visà-vis de la population, que sur le plan externe, vis-à-vis des autres Etats.

Le retour à l'ordre constitutionnel peut, selon Reyntjens (1980), s'effectuer de deux façons: ou bien l'armée se retire de la politique en remettant le pouvoir à des citoyens élus conformément à une nouvelle constitution ou à celle antérieure au coup d'Etat, ou bien les militaires se retirent en leur qualité de militaires, mais conservent le pouvoir dans le cadre d'une nouvelle constitution qui rend leur autorité légitime. Reyntjens parle, dans le premier cas, de "démilitarisation" et, dans le second cas, de "civilisation" (1980: 4). Le processus de démilitarisation peut aboutir au pluripartisme tandis que, dans le second cas, il est pratiquement inévitable d'aboutir à un régime à parti unique sur lequel les militaires peuvent garder leur emprise. Mais, dans l'un et l'autre cas, il faut s'attendre, étant donné la situation actuelle de l'Afrique, à ce que l'armée continue de jouer un rôle actif dans l'arène politique.

\section{LE TOGO}

Depuis son Indépendance, proclamée en 1960, le Togo a connu trois républiques et quatre constitutions, dont la dernière est entrée en vigueur le 31 janvier 1980. La Première République (27 avril 1960-13 janvier 1963) connut deux constitutions: celle du 23 avril 1960, qui entra en vigueur le jour de l'Indépendance (27 avril 1960), et celle du 9 avril 1961. La première constitution instituait un appareil parlementaire et un pouvoir exécutif bicéphale (un chef de l'Etat et un premier ministre), en une chambre de Députés (Prouzet, 1976: 26). "Il s'agissait d'un régime parlementaire rationalisé inspiré par les dispositions de la constitution française de 1958" (Lavroff, 1976:386). Dans la pratique, cependant, le Premier ministre remplissait le role de chef de l'Etat. Le principe d'un pouvoir présidentiel fort, ancré dans cette constitution, fut renforcé par la constitution du 9 avril 1961: le chef de l'Etat, qui devait sa légitimité à des élections directes, nommait les ministres qui étaient directement responsables devant lui. Ce "présidentialisme négro-africain" fut parfois décrit de la façon suivante: "Un Président aussi fort que celui des Etats-Unis, 
un Parlement aussi faible que celui de la Ve République: tels sont les organes creés par la constitution togolaise" (Lavroff,1976).

Face à cet accroissement d'une partie du pouvoir présidentiel, apparut rapidement une opposition de l'élite politique, des militaires et des chef coutumiers. Il s'ensuivit un coup d'Etat, le 13 janvier 1963, qui coûta la vie au chef de l'Etat. Ce coup d'Etat était d'ailleurs une première en Afrique francophone. Ainsi naquit la IIe République, fondée sur la constitution du 11 mai 1963, qui présentait de nombreux points communs avec la constitution française de 1958. Le présidentialisme était maintenu, étant entendu que la fonction de Premier ministre était remplacée par la fonction de vice-Président. La rivalité entre les deux dirigeants suprèmes, le chef de l'Etat et le vice-Président, provoqua une agitation politique et une méfiance mutuelle. Elle s'avéra être la cause du deuxième coup d'Etat, perpétré également le 13 janvier 1967. Le coup d'Etat était dirigé par l'ancien lieutenant-colonel Eyadéma, maintenant général et Président de la République togolaise.

Tout de suite après le coup d'Etat, la constitution fut supprimée, les partis politiques dissous, et il fallait attendre 1979 pour qu'une nouvelle constitution entra en vigueur. Au cours de la période 1967-1979, le pays fut dirigé à l'aide d'ordonnances et de décrets présidentiels. Contrairement à ce que j'ai affirmé plus haut au sujet des régimes militaires, de toute évidence, celui-ci ne sembla pas avoir eu besoin de "légitimer" son pouvoir en lui donnant un fondement constitutionnel. L'apparition de la constitution togolaise de 1979 était étroitement liée à la naissance du parti unique, le Rassemblement du Peuple Togolais (RPT). Au cours d'un congrès tenu en 1979, Eyadéma -souvent désigné aujourd'hui par les termes "l'homme du 13 janvier", en souvenir du 13 janvier 1967 où il prit le pouvoir - avait de nouveau fait savoir qu'il voulait abandonner le pouvoir afin d'offrir la possibilité de mettre en place un régime pluripartiste, comparable à celui que le pays avait connu pendant les deux premières Républiques. La réaction fut la même qu'en 1969 et 1971: le peuple, rassemblé en masse autour du palais présidentiel, demanda qu'Eyadéma resta au pouvoir. Des télégrammes de soutient affluèrent de toutes parts pour l'encourager à rester. Le général accéda au désir du peuple et demeura le chef de l'Etat.

Quelles que furent les véritables motifs de l'annonce de son éventuel retrait, son image de marque et son charisme s'en trouvaient renforcés. Depuis lors, 


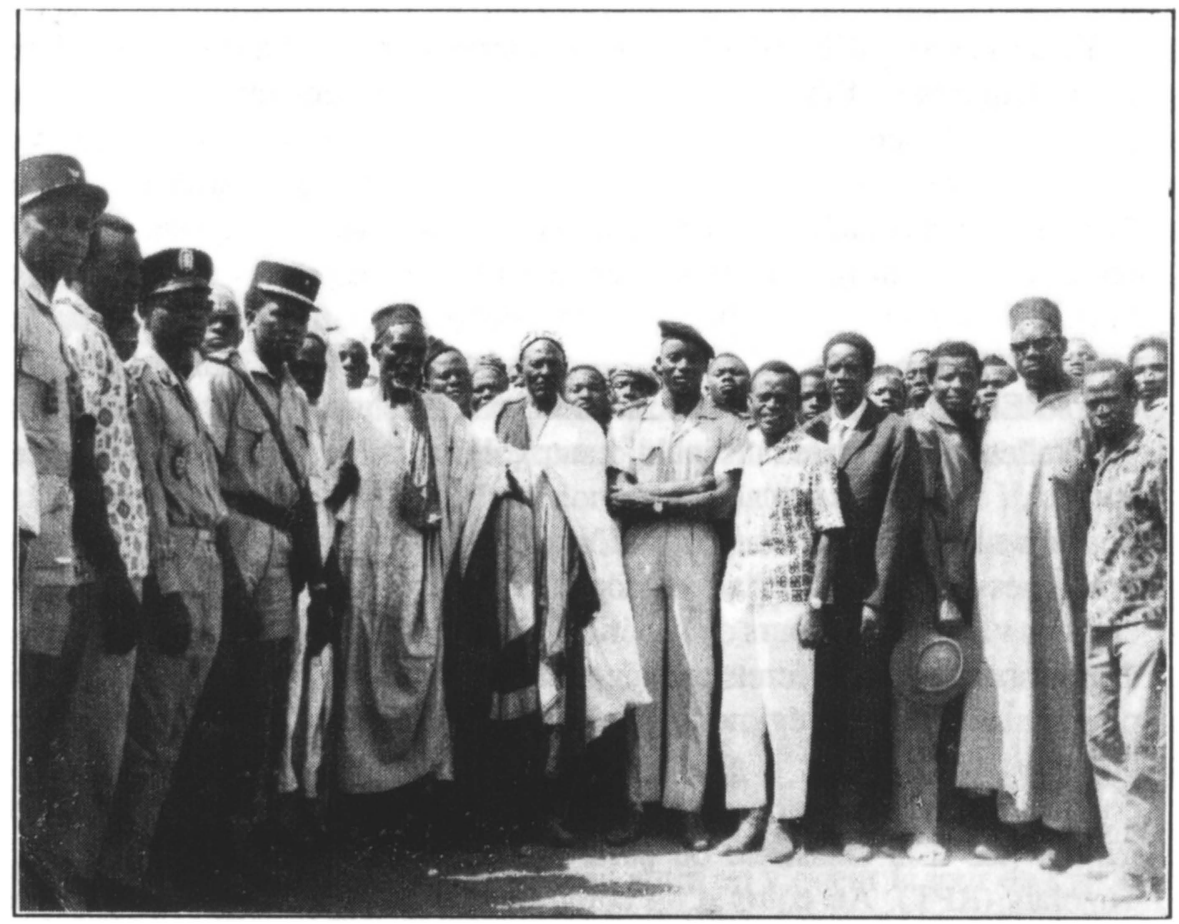

Kapt. Gnassingbé Eyadéma (met baret) in Mango (Noord-Togo) tijdens de officiële benoeming op 13 juni 1963 van Na Tyaba Tyekura (rechts van hem) tot chef supérieur van de Anufom

Foto: EA.B. van Rouveroy van Nieuwaal 
le culte de sa personnalité s'est intensifié, au point même qu'il suscite l'irritation des Togolais eux-mêmes (Harrison Church, 1988:1044). Il est sûr également qu'Eyadéma savait qu'un retour à l'ancien régime des partis était difficilement réalisable à l'époque: tous les partis politiques avaient en effet été dissous en 1967 et il ne resta plus qu' un seul parti, fondé par Eyadéma lui-même.

Le RPT trouva un fondement constitutionnel grâce à une résolution adoptée lors du congrès du parti tenu en 1979, et "demandant" au Bureau politique du RPT d'appliquer une résolution antérieure prévoyant la rédaction d'un modèle de constitution. Ce modèle fut adopté le 30 décembre 1979, comme nous pouvons le lire dans l'en- tête de la constitution. C'est également ce jour-là que le seul candidat à la présidence de la République, Eyadéma, fut rélu chef de l'Etat, avec presque 100\% des suffrages exprimés (Harrison Church, 1988: 1043). La constitution prévoit une Assemblée nationale comprenant à l'époque 67 membres (plus tard, 77), élus pour une durée de cinq ans. Les députés sont tous proposés par le RPT et sont donc à l'origine uniquement destinés à devenir des cadres du parti (Leclerq, 1980: 817).

\section{QUELQUES CARACTÉRISTIQUES DE LA QUATRIÈME CONSTITUTION TOGOLAISE}

Cette constitution ne compte que 53 articles, car "plus les textes sont longs dans nos pays africains, moins ils sont applicables" (Nachaba, 1985). L'essence de cette constitution apparait à l'article 4 du préambule: "Le Rassemblement du Peuple Togolais, parti unique, prime toutes les institutions de l'Etat." Cela signifie qu'au sein de l'Etat togolais, aucun pouvoir n'est supérieur au parti unique. Mais cela implique également que le chef de l'Etat, en tant qu'institution étatique, tombe aussi sous la coupe du parti unique. On peut en douter dans le cas présent car le président actuel, Eyadéma, est aussi "président-fondateur" du parti unique et "père spirituel" des statuts du parti. Ces mêmes statuts sont d'ailleurs primordiaux pour la fonction et le rôle du chef de l'Etat dans l'ensemble de l'appareil de l'Etat et du parti. L'importance des statuts du Rassemblent du Peuple Togolais ressort du chapitre trois de la constitution, article 10, qui y renvoie expressément dans le cadre de la description de la fonction présidentielle . Ainsi est mise en évidence l'intri- 
cation de la constitution elle-même et des statuts du parti unique (Gonidec, 1976, 75-76).

En outre, la constitution prévoit que le président du parti unique est également le seul candidat à la présidence de la République. C'est donc le RPT, et lui seul, qui définit la fonction présidentielle et le contenu a donner à cette fonction, et c'est également le parti qui décide qui accèdera à cette fonction. Le parti unique étant le cerveau chargé de concevoir le développement de l'appareil d'Etat (article 10), il est clair que, dans l'exercice de ses fonctions, le chef de l'Etat devra se conformer a ses idées, s'il ne veut pas perdre le soutien du parti. Mais il pourra également imposer certaines prises de position au sein du parti. Bien que des 53 articles de la constitution, 9 seulement soient consacrés au chef de l'Etat, l'importance que l'on accorde à la place de celui-ci au sein de l'appareil de l'Etat ressort à maintes reprises. Ainsi, le chef de l'Etat réunit trois fonctions:

- il est président du parti unique;

- il est chef du gouvemement, nomme et révoque les ministres (article 20, alinéas 1 et 2);

- il est chef de l'armée togolaise et ministre de la défence.

La concentration des pouvoirs en la personne du chef de l'Etat signifie purement et simplement que celui-ci, malgré un pouvoir exécutif, législatif et judiciaire, supervise et, surtout, domine toutes les institutions de l'Etat. Le chef de l'Etat est donc devenue l'axe autour duquel tout tourne:

"le chef de l'Etat est plutôt dans la situation d'un architecte ou d'un maître d'oeuvre. Il est au centre de tout. C'est lui qui bâtit la Nation, dirige l'Etat et le personnalise à l'extérieur comme à l'intérieur. Dans une large mesure, il se confond avec le système politique lui-même. Il n'en est pas seulement le symbole, mais il le modèle et le controle" (Nachaba, 1985: 27).

Mais nous exprimons quelques réserves sur un point que l'auteur togolais cité ci-dessus se garde bien de soulever, à savoir qu'il ne peut pratiquement pas être question de justice indépendante, bien que l'article 47 essaye de nous faire croire le contraire. L'ampleur du pouvoir du chef de l'Etat togolais ressort encore du fait que le terme constitutionnel de "gouvernment" doit s'entendre comme "cabinet présidentiel": "Il n'y a pas dans la IIIe République Togolaise, de gouvernement qui soit distinct du chef de l'Etat. Celui-ci, chef suprême de l'exécutif, dispose à son gré des ministres, responsables à titre 
exclusif devant lui seul et non en même temps devant l'Assemblée Nationale" (Leclerq, 1980:822).

\section{REMARQUES FINALES}

L' entrée en vigueur de la quatrième constitution n'a, au début, apporté que peu de changements dans la manière de gouverner d'Eyadéma, qui, comme nous l'avons vu, consiste en un présidentialisme très renforcé. Toutefois, en 1985 , on posa les premiers jalons d'un processus de démocratisation en adoptant l'amendement à l'article 24 de la constitution qui permet de proposer des candidats à l'Assemblée Nationale autres que ceux proposés par le RTP (Barbier, 1987; Harrison Church, 1988).

En juin 1987, Eyadéma eut pour la première fois des entretiens avec les responsables des anciens partis politiques, afin de rétablir l'unité nationale qui avait beaucoup souffert des tentatives constantes de coups d'Etat visant à renverser le régime d'Eyadéma. Dans le cadre de la démocratisation, il convient encore de mentionner la création, la même année, d'une commission pour les droits de l'homme.

On irait peut-être trop loin en considérant cette évolution comme une autocritique relative à la foi dans le parti unique. Le processus de démocratisation se limite à quelques actes politiques habiles et peu importants, destinés à juguler l'immobilisme politique et les murmures de mécontentement qui s'élevaient contre la toute-puissance du RPT. Mais il apparut lors d'une conférence tenue en Allemagne fédéral en 1986 sur le thème "Démocratie et parti unique africain", qu'ailleurs également on se pose des questions sur le fonctionnement du régime monopartiste. D'éminents politicologues africains n'hésitèrent pas, eux non plus, à y exprimer des critiques ouvertes. Mengue me Engouang (du Gabon) alla même jusqu'à attaquer carrément le monopartisme: "..., car ce dernier n'admet pas la concurrence et ne tolère pas la contradiction" (1986: 131), et encore: "Il en résulte que dans les régimes africains monopartistes, les parlements ne sont guère représentatifs de toutes les familles politiques. Ils sont plutôt monolothiques, car tous les députés sont issus du même parti" (1986: 133). 
Il semble que le temps est venu ou les chefs africains de partis uniques ne puissent ignorer cette critique. Car il ne leur échappe pas que l'Etat échoue dans de nombreux cas, tant dans le domaine de la gestion politique que dans celui de la justice (van Rouveroy van Nieuwaal, 1988). A cet égard, la situation au Ghana est intéressante. Une étude politicologue effectuée dans ce pays a révélé que dans certaines régions du Ghana, le pouvoir des chefs coutumiers qui a été en veilleuse pendant des années, montre un dynamisme remarquable pour s'adapter aux mutations sociales et politiques (van Rouveroy van Nieuwaal, 1988). Il n'est pas à écarter que ce pouvoir jouera un rôle déterminant dans les tentatives futures de transformations socio-économiques au niveau régional et national. Rawlings, le dirigeant actuel du Ghana, a dâ se rendre à cette évidence (Neue Zârcher Zeitung, 8.01.89, p.5). L'Etat togolais reconnaît également que les chefs traditionnels peuvent servir d'intermédiaires dans les rapports entre les autorités et le peuple. Car la pratique a appris aux autorités que les chances de réussite des projets d'innovation et de développement augmentent lorsque les chefs coutumiers y participent activement.

Pour l'instant, le pouvoir central, ou plutôt le RPT, n'en a pas encore tiré la conclusion que les chefs traditionnels ou néo-traditionnels devaient être investis de pouvoirs plus grands que ceux qui leur sont actuellement impartis en vertu des règlements coloniaux, malgré les fréquents échecs de l'appareil administratif et judiciaire inspiré du modèle occidental, ou plutôt français. Une reconnaissance plus large de l'autorité des chefs coutumiers pourrait certainement encourager le citoyen à participer plus activement à la gestion politique et à la justice, et favoriser le développement de la démocratie sur des bases purement africains, même si les pouvoirs du parti unique s'en trouvent réduits. 


\section{REMERCIEMENTS}

Je remercie Atsu Koffi Améga (Cour Suprême, Lomé, Togo), J-Cl. Barbier (ORSTOM, Lomé, Togo), P. Van Damme (RU-Gent), D. Beke (RU-Gent) et T. von Trotha pour leur critique et Mme C. Miginiac pour la traduction en français aussi fidèle que possible. Néanmoins j'assume toute la responsabilité pour l'erreur occasionnel quelconque.

\section{BIBLIOGRAPHIE SOMMAIRE}

Barbier, J-Cl., Jalons pour une sociologie électoral du Togo:1958-1985 in: Politique Africaine, no. 27: 6-18.

Bechenane, M., Les Coups d'Etat en Afrique, 1983, Publisud: Paris.

Colement, J.S.\& Rosberg, G., African One Party Systems and Modernization, in: Political Modemization, 1971, C.E. Welch ed., Belmont, Ca.

Doombos, M.e.a., Constitutional Form and Ideological Content: the Preambles of French Language Constitutions in Africa, 1984: 41-100, in: Aspekten van Staat en Maatschappij in Afrika , W.v.Binsbergen \& G. Hessling eds. Research Report no. 22, Afrika- studiecentrum: Leyde.

Fernandes Mendes, H., Onafhankelijkheid en parlementair stelsel in Suriname, 1989, thèse universitaire, Leyde, Tjeenk Willink: Zwolle, Pays - Bas.

Goldschmid, J., Constitutioneel Pluralisme: enkele opmerkingen en vraagtekens, 1984: 29-40, in: Aspekten van Staat en Maatschappijin Afrika, W.v.Binsbergen \& G. Hesseling eds. Research Report no. 22, Afrika-studiecentrum: Leyde.

Gonidec, P.F., Les Droits Africains, 1976, Librairie générale de droit et de jurisprudence: Paris.

Gonidec, P.F., A quoi servent les constitutions africaines? Réflections sur le constitutionalisme africain, in: Revue Juridique et Politique. Indépendance et Coopération, no. 4, 1988: 849-866.

Harrison Church, R.J., Togo, in: Africa. South of the Sahara, pp. 1042-1056, 18e édition, 1988, Europa Publications: Londres.

Hesseling, G., Histoire politique du Sénégal - institutions, droit et société, 1985, Karthala et ASC: Paris et Leiden, Pays - Bas. 
Kamto, M., Pouvoir et Droit en Afrique Noire, 1987, Librairie général de droit et de jurisprudence: Paris.

Kirk-Green, A.H.M., A Sense of Belonging: the Nigerian Constitution of 1979 and the Promotion of National Loyalty, in: Journal of Commonwealth \& Comparative Politics, vol. 26, no. 2, 1988: 158-172.

McGregor Hutcheson, A. Botswana, in: Africa South of the Sahara, pp.268-284, 18e édition, Europa Publications, Londres.

Jackson, R.H. \& Rosberg, C.G., The marginality of African States, in: African Independence - the first 25 years, G.M. Carter \& P.O'Meara eds. 1985: 45-70, Indiana University Press: Bloomington, Etats-Unis.

Lavroff, D.G., Les Systèmes constitutionnels en Afrique Noire - les Etats francophones, 1976, Pedone: Paris.

Leclerq, C.L., La constitution togolaise du 13 janvier 1980, in: Revue Juridique et Politique. Politique et Coopération, 1980, volume 34, no. 4: 818-824.

Luckman, R., Political and Social Problems of Development, in: Africa South of the Sahara, pp. 50-62, 18e édition, Europa Publications, Londres.

Menque me Engouang, F. Parlementarisme et monopartisme en Afrique noire francophone, in: Recueil Penant 1986, no. 790-791: 129-140.

Moore, S.F., Law as Process - an Anthropological Approach, 1978, Routledge \& Kegan Paul: London.

Nachaba Ouatara Fambaré, Le régime constitutionnel au Togo depuis 1979, in: RecueilPenant, 1985 , no. 786.

Nwabueze, B.O.: Constitutionalism in Emergent States, 1973, C. Hurst: Londres.

Prouzet, M., La république du Togo, 1976, Encyclopédie Politique et le Constitutionnelle, série: Afrique; Editions Berger-Levrault, Paris.

Read, J.S. The new Constitution of Nigeria, 1979: "the Washington Model"? in: Џoumal of African Law, 1979: 131-169.

Reyntjes, F., Off the Horseback? Legal Aspects of the Return to Constitutional Government in Africa South of the Sahara, 1980,in: Verfassung und Recht in Uebersee, Beiheft 10, Nomos Verlagsgeselschaft: Baden Baden.

Reyntjes, F., Recent Developments in the Public Law of Francophone African States, in: Joumal of African Law, vol.30, automne 1986, no. 2: 75-90. 
Rouveroy van Nieuwaal, van E.A.B., Etat et Pouvoir Traditionnel en Afrique: position ambiguë du Chef coutumier face à l'Etat Africain, in: Droit et Cultures, no. 15/16, 1988: $71-115$.

Rouveroy van Nieuwaal, van E.A.B., Sorcellerie et la Justice Coutumière au Togo: une quantité négligeable? in: Joumal of Legal Pluralism, à paraître, 1989.

Sklar, R.L., The Colonial Imprint on African Political Thought, 1985: 1-30, in: African Independence - the first 25 years, G.M. Carter \& P. O'Meara eds., Indiana University Press: Bloomington, Etats-Unis.

Trubek, D., Toward a Social Theory of Law: an Essay on the Study of Law and Development, in: Yale Law Joumal, 1972, 82: 5. 